\title{
Braquiópodes (Rhynchonelliformea, Bouchardioidea) neógenos da bacia de Pelotas (RS) e seu significado paleoambiental
}

\author{
Marcello Guimarães Simões ${ }^{1}$, Suzana Aparecida Matos da Silva ${ }^{2}$, \\ Sabrina Coelho Rodrigues ${ }^{3}$ \& João Carlos Coimbra ${ }^{4}$
}

\begin{abstract}
Resumo A ocorrência de braquiópodes no Neógeno da Bacia de Pelotas é conhecida desde 1862. A despeito disso, porém, estudos detalhados de sistemática e tafonomia ainda não existem. Investigações conduzidas meio século atrás indicaram que os braquiópodes poderiam ser atribuídos à espécie Bouchardia cf. zitteli, comum à Formação San Julian, Oligoceno Superior, Argentina. A revisão realizada indica, contudo, que a espécie presente é Bouchardia transplatina. Na porção uruguaia da Bacia de Pelotas, B. transplatina é conhecida na Formação Camacho, Mioceno. Em adição, pequenas conchas de braquiópodes foram recuperadas também de material de calha, de poços Petrobras (2PJ-1-RS, 2PN-1-RS e 2GA-1-RS), na porção continental da Bacia de Pelotas. Esses braquiópodes provêm de intervalo estratigráfico (130 a 150 metros) correspondente à Zona Henryhowella evax (Mioceno). A despeito do alto grau de dissolução das conchas, essas podem ser atribuídas, sem dúvida, a Bouchardia sp. Os dados acima são relevantes, pois: 1- Bouchardia possui afinidades com águas quentes (tropicais/subtropicais). Presentemente, formas viventes desse gênero habitam águas em latitudes de até $34^{\circ} \mathrm{S}$, mas a maior abundância está em torno de $23^{\circ} \mathrm{S}$; 2- embora ocorram em profundidades superiores a 200 metros, Bouchardia rosea (espécie vivente) é mais abundante em águas plataformais rasas, ricas em nutrientes; 3- a presença de Bouchardia, no Mioceno da Bacia de Pelotas indica que, pelo menos no intervalo da Zona Henryhowella evax, condições de águas quentes relacionadas à Corrente do Brasil, prevaleceram. Essa interpretação está de acordo com dados paleoeoceanográficos e paleoclimáticos, fornecidos por ostracodes e foraminíferos que co-ocorrem com os braquiópodes estudados.
\end{abstract}

Palavras-chave: Brachiopoda, Bouchardia, Mioceno, bacia de Pelotas.

\begin{abstract}
Neogene brachiopods (Rhynchonelliformea, Bouchardioidea) from the Pelotas basin $(R S)$ and their paleoenvironmental significance. The occurrence of brachiopods in Cenozoic rocks of the Pelotas Basin is known since 1862. In spite of that, detailed systematic and taphonomic studies are still missing. Investigations made a half century ago, have suggested that these brachiopods could belong to Bouchardia cf. zitteli, a species found in the San Julian Formation, Late Oligocene, Argentina. Our data suggest that those brachiopods may resemble Bouchardia transplatina. In the Uruguayan portion of the Pelotas Basin B. transplatina is known in rocks of the Camacho Formation, Miocene. In addition, small recrystallized shells of brachiopods were also recovered from three Petrobras boreholes (2PJ-1-RS, 2PN-1-RS, and 2GA-1-RS) from the Pelotas Basin. Brachiopods come from the interval of 130 to 150 meters within the Miocene Henryhowella evax Zone. Despite the degree of taphonomic modification of those brachiopod shells they indubitably belong to Bouchardia sp. This is noteworthy for various reasons: 1- Bouchardia is a brachiopod with warm water affinities. Presently, extant members of this genus are unknown in latitudes up to $34^{\circ} \mathrm{S}$, with the main records at $23^{\circ} \mathrm{S}$. 2- Although occurring in depths down to 200 meters, the living member (Bouchardia rosea) of this genus is most abundant in shallow platformal, nutrient-rich waters. 3- The occurrence of Bouchardia in the Miocene of the Pelotas Basin indicates that, at least to the interval of Henryhowella evax Zone, warm waters of the Brazilian currents prevail. This interpretation is in strong accordance with other paleoeoceanographic and paleoclimatic data offered by various groups of co-occurring microfossils, such as ostracodes and foraminifers.
\end{abstract}

Keywords: Brachiopoda, Bouchardia, Miocene, Pelotas basin.

INTRODUÇÃO Embora a presença de braquiópodes rinconeliformes, na sucessão miocênica da Bacia de Pelotas, seja conhecida desde 1862 (White 1908), esses fósseis nunca foram objeto de estudos detalhados.
De fato, a pesquisa paleontológica conduzida na Bacia de Pelotas vem, sistematicamente, enfocando os mais diversos grupos de microfósseis (Tab. 1). Porém, os dados disponíveis para outros grupos, tais como os mi-

1 - Departamento de Zoologia, UNESP/IBB, Botucatu (SP), Brasil. E-mail: btsimoes@ibb.unesp.br

2 - Programa de Iniciação Científica em Ciências Biológicas, Departamento de Zoologia, UNESP/IBB, Botucatu (SP), Brasil. E-mail: sumatos.s@gmail.com

3 - Departamento de Zoologia, UNESP/IBB, Botucatu (SP), Brasil. E-mail: scoelho@ibb.unesp.br

4 - Departamento de Paleontologia e Estratigrafia, UFRGS/IGEO, Porto Alegre (RS), Brasil. E-mail: joao.coimbra@ufrgs.br 
Tabela 1- Relação dos principais estudos com microfósseis da Bacia de Pelotas.

\begin{tabular}{l|l|l}
\hline Microfósseis & Autor(es) & Ano \\
\hline Foraminíferos & Closs & 1967 \\
Palinomorfos & Daemon & 1969 \\
Foraminíferos & Closs & 1970 \\
Foraminíferos & Fernandes & 1975 \\
Foraminíferos & Thiesen & 1975 \\
Ostracodes & Sanguinetti & 1979 \\
Ostracodes & Sanguinetti & 1980 \\
Foraminíferos, Radiolários & Madeira-Falcetta et al. & 1980 \\
Nanofósseis Calcários & Gomide & 1989 \\
Ostracodes & Sanguinetti, Ornellas \& Coimbra & 1991 \\
Ostracodes & Sanguinetti, Ornellas, Coimbra \& Ramos & 1992 \\
Ostracodes & Carreño, Coimbra \& Sanguinetti & 1997 \\
Ostracodes & Carreño, Coimbra \& Carmo & 1999 \\
Foraminíferos & Coimbra, Carreño \& Anjos-Zerfass & in press \\
\hline
\end{tabular}

cro-moluscos e micro-braquiópodes são ainda escassos (Sanguinetti 1980) ou, em alguns casos, inexistentes.

Com exceção de uma nota curta de Martins (1952), que examinou as rochas e os fósseis da chamada "Sondagem de 1862", as informações a respeito da presença de braquiópodes fósseis nos estratos da Bacia de Pelotas, restringem-se a citações breves (vide, por exemplo, Gliesch 1932, Closs 1967, 1970, Sanguinetti 1980). Entretanto, a presença de braquiópodes atribuíveis ao gênero Bouchardia, na Bacia de Pelotas, aventada por Martins (1952) é, por várias razões, cientificamente instigante. Em primeiro lugar, os bouchardídeos são importantes para biocorrelação e paleoceanografia (vide discussão em Manceñido \& Griffin 1988), sendo que na América do Sul, os registros estratigráficos de espécies desse gênero estendem-se até o limite Cretáceo/ Paleoceno. Ao longo do Cenozóico as espécies de Bouchardia e formas afins mostram nítido deslocamento em direção às águas mais quentes ao norte (Ihering 1903, p. 341, Manceñido \& Griffin 1988). Em segundo lugar, ao descrever e ilustrar os braquiópodes fósseis da "Sondagem de 1862", Martins (1952, p. 4) atribuiu as formas presentes na Bacia de Pelotas à espécie Bouchardia cf. zitteli. Esse fato chama atenção, porque as camadas contendo Bouchardia, na Bacia de Pelotas, são, ao que tudo indica, miocênicas (vide Sanguinetti 1980 e Closs 1970). Do mesmo modo, as camadas coevas da Bacia del Leste, Uruguai, contendo acumulações densas de conchas de Bouchardia são também referíveis ao Mioceno (Martinez 1994). Entretanto, Bouchardia zitteli é conhecida apenas para estratos oligocênicos da Formação San Julián, da Patagônia, Argentina (del Rio 2004).

No contexto acima, seria interessante re-exa- minar os braquiópodes da Bacia de Pelotas, com a finalidade de determinar qual a espécie do gênero Bouchardia está presente nos estratos miocênicos. Assim sendo, os objetivos gerais da presente contribuição são os de ampliar os conhecimentos relativos à sistemática e distribuição estratigráfica dos braquiópodes fósseis da Bacia de Pelotas, discutindo suas implicações paleoceanográficas. Para cumprir esse objetivo foram re-examinadas(os): (a) as amostras de calha dos poços perfurados pela Petrobras, na porção continental da Bacia de Pelotas; (b) as amostras do testemunho de "Sondagem de 1862" (Martins 1952), depositadas no Museu Nacional do Rio de Janeiro, RJ e (c) os dados de literatura, em especial das seções estudadas por Sanguinetti (1980) e o material figurado por Martins (1952), cuja qualidade das ilustrações permite a identificação precisa dos espécimes.

\section{MATERIAL M MÉTODOS}

Área de estudo A margem continental da América do Sul, a partir de seu extremo meridional, inicia com um extenso trecho retilíneo, orientado a NE-SW, em que se inclui a Bacia de Pelotas (Milani et al. 2000). No extremo nordeste deste trecho desenvolve-se a Dorsal de São Paulo, cujo prolongamento para NW, no sentido do continente, encontra o Arco de Ponta Grossa. Esta feição corresponde a um braço abortado do rifte sul-atlântico inteiramente dominado por magmatismo básico do Eocretáceo (134-128 Ma) que afeta os pacotes sedimentares paleozóicos da Bacia do Paraná e as regiões de embasamento vizinhas (Milani et al. 2000).

A Bacia de Pelotas, situada no extremo sul da margem continental brasileira, com uma área de cer- 
ca de $250.000 \mathrm{~km}^{2}$, até a isóbata de 3.000 metros e orientação geral NE-SW (Milani et al. 2000, Mohriak 2003), tem seu preenchimento sedimentar representado por três megasseqüências, que materializam as principais fases de evolução tectônica da bacia (Dias et al. 1994, Fontana 1996, Mohriak 2003). A sucessão onde se encontram os fósseis aqui estudados pertence a megasseqüência pós-rifte, representativa de sedimentação marinha na bacia (Dias et al. 1994, Mohriak 2003). Essa megasseqüência é dividida em cinco seqüências deposicionais (Dias et al. 1994), sendo que os braquiópodes parecem ocorrer na quinta seqüência, que inclui, predominantemente, pelitos, com raras intercalações de arenitos turbidíticos, do Oligoceno ao Holoceno (Dias et al. 1994, Mohriak 2003).

Procedência dos espécimes examinados Para o presente estudo foram examinadas lâminas provenientes de amostras de calha das sondagens 2-CA-1-RS (Curral Alto), 2-PJ-1-RS (Ponta do Juncal), 2-CL-1-RS (Cassino), 2-PN-1-RS (Povo Novo), 2-GA-1-RS (Graxaím), 2-GA-2-RS (Graxaím), 2-MO-1-RS (Mostardas) e 2-PS-1-RS (Palmares do Sul) (Figs. 1, 2), perfuradas pela Petrobras, no período de 1958-1964. Conchas de braquiópodes foram recuperadas de amostras dos poços 2-PN-1-RS (Povo Novo) e 2-GA-1-RS (Graxaím) (Figs. 1, 2). Closs (1970) e Sanguinetti (1980) registraram também a presença de conchas de braquiópodes no poço 2-PJ-1-RS (Ponta do Juncal) (Figs. 1, 2). Todo material examinado pertence ao Laboratório de Microfósseis Calcários, do Departamento de Paleontologia e Estratigrafia, do Instituto de Geociências, da Universidade Federal do Rio Grande do Sul.

Além disso, foram examinadas também réplicas das conchas originais preservadas nos testemunhos da "Sondagem de 1862", estudados por Martins (1952) (Fig. 3). Os originais desse material estão depositados no Museu Nacional no Rio de Janeiro, sob o número MN 5065-I e as réplicas na Coleção Científica do Departamento de Zoologia do IBB/UNESP, sob o código DZP.

Adicionalmente, para fins comparativos foram examinadas conchas de Bouchardia zitteli $(\mathrm{n}=80)$, provenientes da Formação San Julián, Patagônia, Argentina, bem como de Bouchardia transplatina $(\mathrm{n}=135)$, coletadas na Formação Camacho, Uruguai. Tais conchas pertencem às coleções científicas do Instituto de Geociências, Universidade de São Paulo e Instituto Nacional de Geologia y Paleontologia, Facultad de Ciências, Uruguai, respectivamente.

Triagem dos espécimes provenientes do material de calha e microscopia eletrônica de varredura As lâminas estudadas, que contêm os braquiópodes provenientes dos poços Petrobras, resultaram da triagem de ostracodes e foraminíferos para os estudos de Sanguinetti $(1979,1980)$, dentre outros. As lâminas já estavam separadas por intervalos estratigráficos. Portanto, os interessados devem se referir a Sanguinetti (1979, 1980) para os métodos de preparação e triagem desse material. As lâminas resultantes dos estudos acima

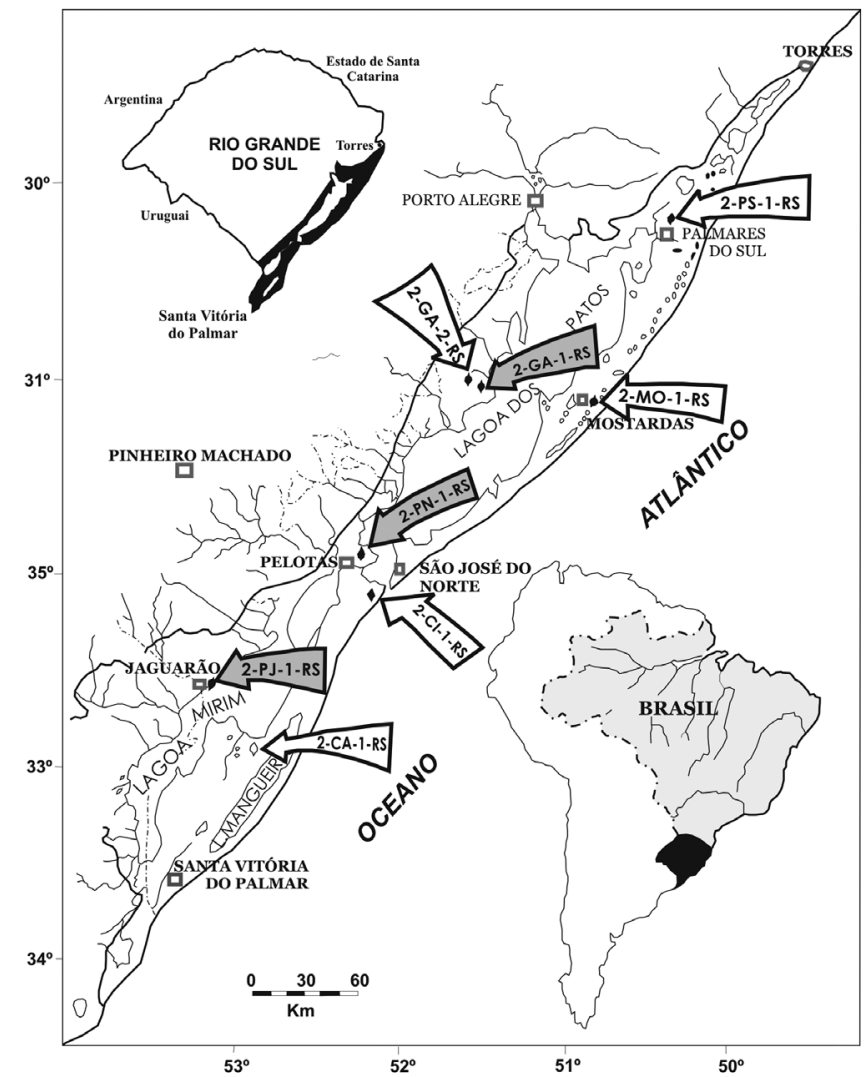

Figura 1 - Localização dos poços Petrobras, na porção continental da Bacia de Pelotas, Rio Grande do Sul. Em destaque os poços que contêm material de calha, com conchas de braquiópodes. Modificado de Sanguinetti (1980).

mencionados contêm ainda mistura de conchas de moluscos, carapaças de briozoários, além de outros elementos bioclásticos. Para a triagem das conchas de braquiópodes, as lâminas contendo o material bioclástico, foram organizadas por poço e intervalo estratigráfico. $\mathrm{O}$ material fóssil presente nas lâminas foi examinado sob estereomicroscópio.

Após a triagem, os espécimes de braquiópodes encontrados nas lâminas disponíveis para estudo foram submetidos à Microscopia Eletrônica de Varredura, no Instituto de Biociências/Unesp, Botucatu, SP, sendo que as imagens obtidas foram utilizadas para a descrição e a identificação taxonômica dos elementos presentes.

\section{RESULTADOS}

\section{Paleontologia sistemática}

Filo Brachiopoda Duméril 1806

Subfilo Rhynchonelliformea Williams, Carlson,

Brunton, Holmer \& Popov 1996

Classe Rhynchonellata Williams, Carlson, Brunton,

Holmer \& Popov 1996

Ordem Terebratulida Waagen 1883

Superfamília Bouchardioidea MacKinnon \& Lee 2006

Família Bouchardiidae Allan 1940

Gênero Bouchardia Davidson 1850 


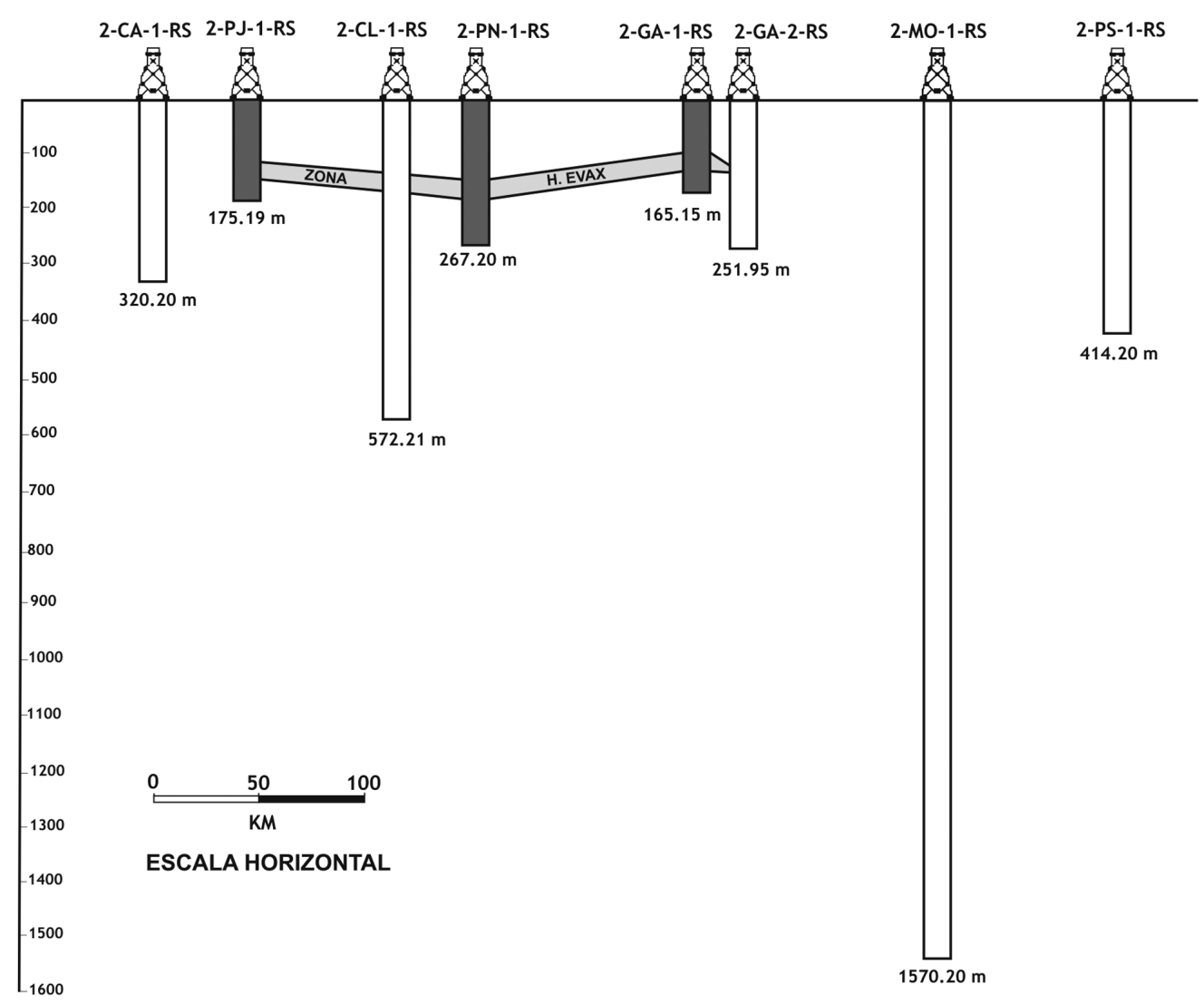

Figura 2 - Distribuição da Zona Henryhowella evax (Mioceno) e, em destaque, os poços que contêm braquiópodes. Modificado de Sanguinetti (1980).

\section{Espécie-tipo Bouchardia rosea Mawe 1823}

Outras espécies Bouchardia zitteli Ihering 1897, Bouchardia conspicua Feruglio 1823, Bouchardia transplatina Ihering 1907, Bouchardia antarctica $\mathrm{Bu}-$ ckman 1910, Bouchardia exigua Camacho 1949.

DISCUSSÃO Conforme demonstrado por Mello (2004), a diferenciação entre as espécies de Bouchardiidae não é simples. Não surpreende, portanto, que Martins (1952), com base em poucos exemplares e reduzida base bibliográfica (vide comentários do autor à página cinco do referido artigo), tenha atribuído os exemplares da Bacia de Pelotas, a Bouchardia cf. zitteli. Ao que tudo indica Manceñido \& Griffin (1988) foram os primeiros autores a discordarem dessa interpretação. Martins (1952) atribuiu os espécimes de braquiópodes da "Sondagem de 1862", à espécie Bouchardia cf. zitteli, fundamentado na presença de um "braquídio caloso" (= sem ramificação) nos espécimes examinados. Segundo Martins (1952, p. 4), a diferenciação entres espécies de Bouchardia poderia ser feita com base nessa feição. $\mathrm{O}$ autor comentou que, devido ao aparelho braquial, caloso e simples, os espécimes da "Sondagem de 1862", diferem "da Bouchardia rosea (Mawe), atual, e da Bouchardia transplatina Ihering, do Entrerriano da Argentina, que o têm ramificado, em âncora; pelo mesmo caráter, assemelha-se à B. patagonica Ihering, do Salamanqueano e à $B$. zitteli Ihering, do Patagoniano da Argentina" (Martins 1952, p. 4).

Em primeiro lugar, é preciso esclarecer que a presença ou ausência de braquídio simples, ou seja, sem ramos ascendentes não é caráter diagnóstico de Bouchardia. A presença ou ausência dessa estrutura (ramos do braquídio junto ao septo), nas conchas fósseis e atuais, recém mortas, deve-se a problemas de preservação. Todo Bouchardioidea possui braquídio incompleto, com ramos ascendentes separados e suspensos na cavidade do manto (Fig. 4). Mesmo nas formas viventes e com conchas ainda articuladas, os ramos do braquídio podem ser perdidos, dada à fragilidade dessa estrutura. Em segundo lugar, conforme ilustrado na figura 5, o braquídio de espécimes bem preservados de Bouchardia rosea (vivente) e Bouchardia zitteli (Oligoceno), possuem ambos ramos ascendentes. Existe menção ainda da presença de braquídio incompleto para as formas Bouchardia antarctica (Eoceno-Oligoceno) e Bouchardia transplatina (Mioceno) (Mello 2004). Outro aspecto importante relacionado ao braquídio de Bouchardioidea é o fato de que os ramos ascendentes não estão presentes em todos os estágios ontogenéticos da concha. Em geral, há gradual desenvolvimento dos ramos ascendentes, a partir das conchas com dimensão igual ou superior a $2 \mathrm{~mm}$ (Mello 2004). 


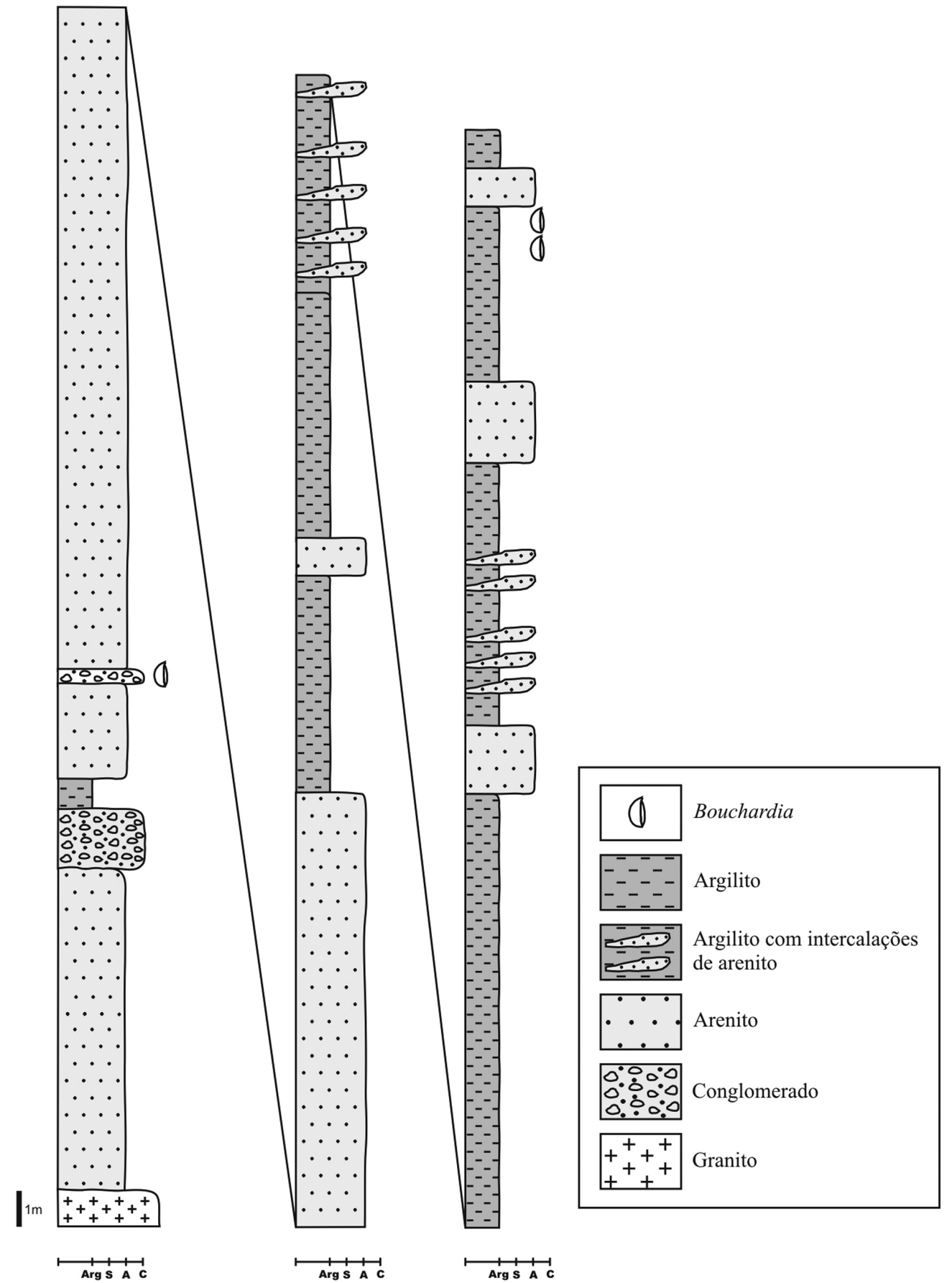

Figura 3 - Seção simplificada, do poço perfurado por Ângelo Cassapi, "Sondagem de 1862”, em Pelotas, Rio Grande do Sul. Dados extraídos de White (1908). Note os horizontes contendo conchas de Bouchardia transplatina.

Em conclusão, portanto, fundamentar a diagnose de conchas do gênero Bouchardia nos ramos ascendentes do braquídio não é procedimento correto. Os espécimes da "Sondagem de 1862" possuem todos mais de $2 \mathrm{~mm}$ de comprimento e estão desarticulados, com claros sinais de dissolução. Dessa forma, a ausência dessa estrutura (ramos do braquídio) deve-se, provavelmente, a fatores bioestratinômicos e diagenéticos.

Bouchardia transplatina Ihering 1907 (Fig. 5)
LOCALIDADE Os espécimes aqui referidos à espécie Bouchardia transplatina são provenientes da "Sondagem de 1862", realizada por Ângelo Cassapi, em Pelotas, Rio Grande do Sul, Brasil.

MATERIAL Três espécimes, duas valvas dorsais e uma valva ventral, todas réplicas dos originais pertencentes ao Museu Nacional. Esses espécimes estão depositados na coleção paleontológica, do Departamento de Zoologia do IBB/UNESP, código: DZP 470, 471, 472. 


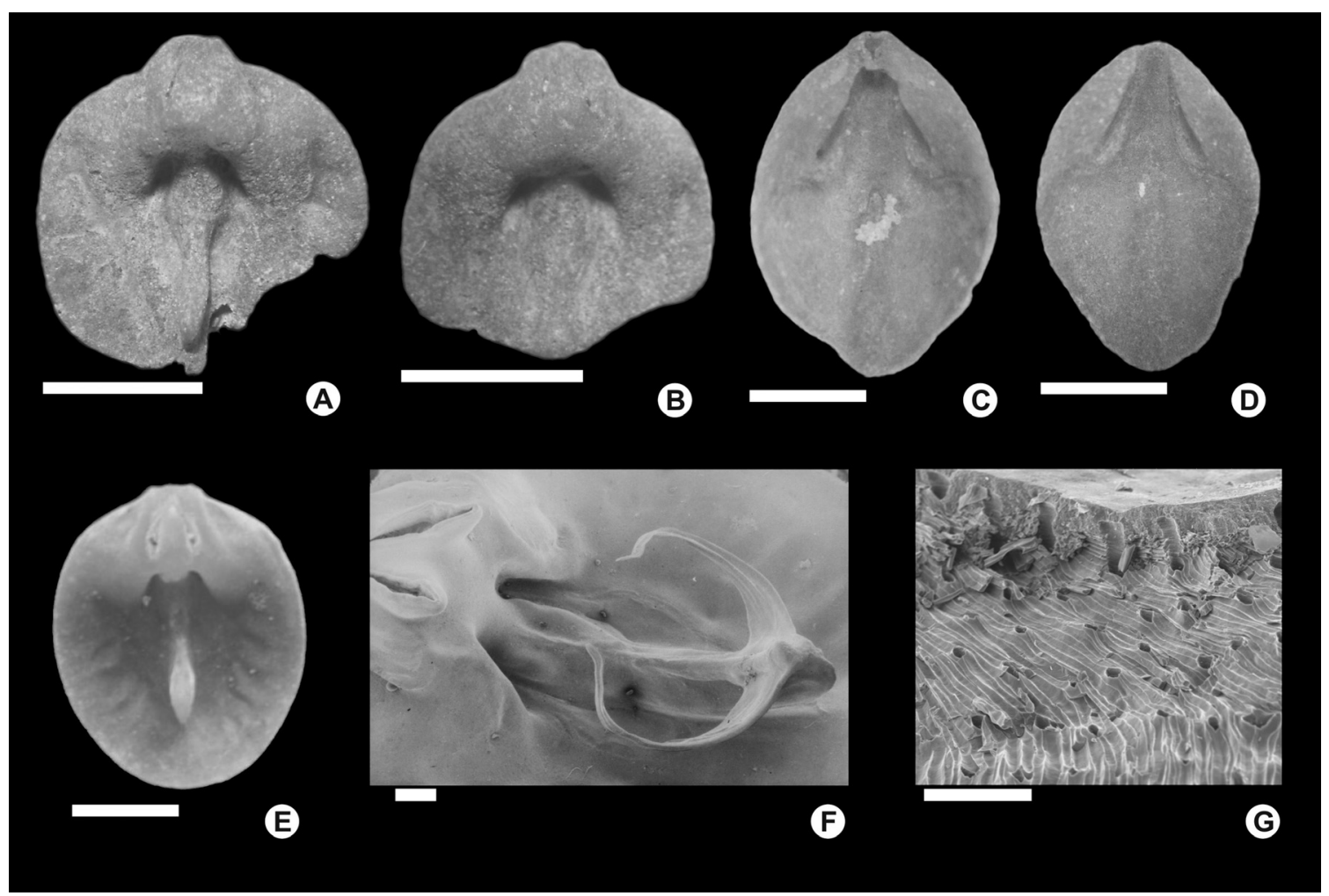

Figura 4 - Espécimes de Bouchardia rosea, coletadas na costa norte do Estado de São Paulo. A, $B$-Valvas dorsais, mostrando arredondamento das regiões mais proeminentes da concha, assim como superficie com textura granular, por dissolução, espécimes DZP-18681 e DZP-18682. C, DValvas ventrais mostrando diferentes graus de desgaste da charneira, por dissolução, espécimes DZP-18683 e DZP-18684. E-Valva dorsal, sem alterações tafonômicas, porém com os ramos do braquídio junto ao septo ausentes, espécime DZP-18670. F-Valva dorsal, sem alterações tafonômicas. Note os ramos do braquidio junto ao septo, espécime DZP-18685. G- Detalhe de valva dorsal, em corte transversal, mostrando as endopontuações e as fibras da camada secundária da concha, espécime DZP- 18685. Vide texto para discussão. Escala gráfica: $5 \mathrm{~mm} \mathrm{em} \mathrm{A,} \mathrm{B,} \mathrm{C,} \mathrm{De}$ E; $1 \mathrm{~mm} \mathrm{em} \mathrm{F,} 100$ нт ет $G$.

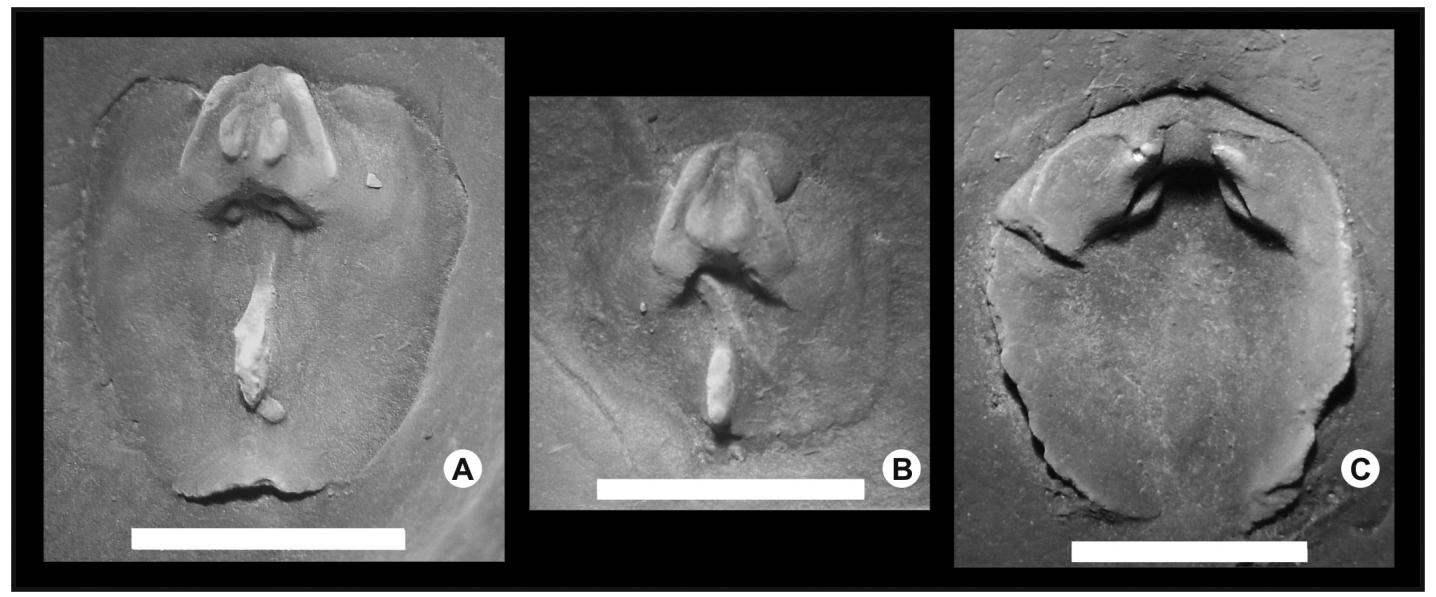

Figura 5 - Conchas de Bouchardia transplatina, "Sondagem de 1862". A-Valva dorsal, desarticulada, mostrando desgaste da região da charneira e septo mediano, por dissolução, espécimes DZP-407. B-Valva dorsal, desarticulada, com a margem da concha dissolvida. Note o arredondamento da estrutura da charneira, espécime DZP-471. C-Valva ventral, com a charneira bem preservada, mas com as margens dissolvidas, espécime DZP-472. Discussão no texto. Escala gráfica: $5 \mathrm{~mm}$. 
DISCUSSÃO Conforme ilustrado na figura 4, as conchas da "Sondagem de 1862" apresentam contorno ligeiramente curvilíneo, com alongamento antero-posterior e margens curvas. Tal característica é típica de Bouchardia transplatina, pois em Bouchardia zitteli a forma da concha é poligonal, com as margens retas. A comissura anterior da valva dorsal, do espécime ilustrado por Martins (1952) é claramente côncava o que denota a presença de uma dobra, quando ambas as valvas estão articuladas, ou seja, a concha é unisulcada. Essa feição é bem marcada em Bouchardia transplatina e, na forma vivente, Bouchardia rosea (Mello 2004). Já as conchas de Bouchardia zitteli mostramse, por sua vez, quase que rectimarginadas. Nas ilustrações de Marins (1952), a valva ventral mostra uma nítida carena, ou seja, uma elevação angular da superfície externa da concha. Essa elevação estende-se antero-posteriormente junto à linha média da valva, conferindo convexidade à concha (vide Mello 2004, p. 39). Em Bouchardia zitteli a convexidade da concha é menor do que a de Bouchardia transplatina, cuja superfície da valva ventral é caracterizada pela presença da carena. Em realidade, as conchas de Bouchardia transplatina são convexas, enquanto as de Bouchardia zitteli são ligeiramente plano-convexas.

Em síntese, as conchas provenientes da "Sondagem de 1862" são aqui atribuídas a Bouchardia transplatina, uma vez que não mostram algumas das principais feições diagnósticas de Bouchardia zitteli (vide Mello 2004, p 36.). Essa interpretação corrobora a suposição de Manceñido \& Griffin (1988), para os quais os espécimes da Bacia de Pelotas não pertenceriam à espécie Bouchardia $\mathrm{cf}$. zitteli, mas possivelmente a Bouchardia transplatina.

\section{Bouchardia sp. (Figs. 6, 7)}

LOCALIDADE Os espécimes aqui referidos à espécie Bouchardia sp. são provenientes de amostras de calha das sondagens 2-PN-1-RS (Povo Novo) e 2-GA-1-RS (Graxaím), cujos poços foram perfurados pela Petrobras, no período de 1958-1964, Rio Grande do Sul, Brasil.

MATERIAL Dois espécimes de valvas articuladas, fechadas e completas, recuperadas no poço 2-PN-1RS (Povo Novo) (DZP-18674, 18675); dois espécimes fragmentados de valvas dorsais (DZP-18679, 18680) e três espécimes fragmentados (uma valva ventral, DZP18676, e duas indeterminadas, DZP-18677, 18678), provenientes do poço 2-GA-1-RS (Graxaím).

DISCUSSÃO As alterações tafonômicas presentes nas conchas dos braquiópodes provenientes do material de calha (poços 2-PN-1-RS e 2-GA-1-RS) não permitem identificação ao nível específico, essas, porém podem ser indubitavelmente atribuídas ao gênero Bouchardia. Algumas características diagnósticas desse gênero, tais como o processo cardinal bilobado e os dentes e fossetas em "V", na valva dorsal, estão presentes no espécime DZP-18679, proveniente dos poços 2-GA-1-

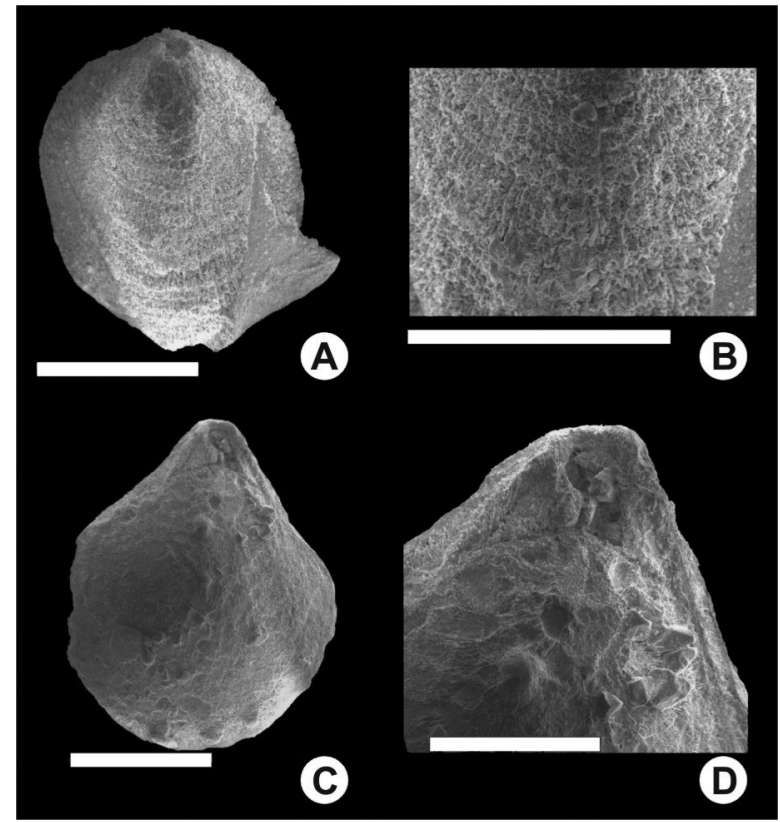

Figura 6 - Espécimes de Bouchardia sp., provenientes do poço 2-PN-1-RS. A-Vista ventral de espécime articulado, mostrando a superficie da concha, com textura granular, por dissolução da calcita, espécime DZP-18674. B- Detalhe do mesmo exemplar, ressaltando a textura granular da superficie da concha. $C$-Vista dorsal de espécime articulado, com destaque para a recristalização da concha, espécime DZP18675. D-Detalhe da superficie do mesmo exemplar. Vide discussão no texto. Escala gráfica: $500 \mu \mathrm{m} \mathrm{em}$ A, $300 \mu \mathrm{m} \mathrm{em} \mathrm{B,} 2 \mathrm{~mm} \mathrm{em} C, 1 \mathrm{~mm} \mathrm{em} D$.

RS (Graxaím). Com relação aos fragmentos de valvas dorsais, alguns possuem a região posterior preservada. Nesses exemplares, o processo cardinal bilobado, típico de Bouchardia está bem preservado. As alterações presentes, conforme já visto, dizem respeito à dissolução da concha. Há também vestígios dos sulcos em $\mathrm{V}$ invertido (Fig. 7a). Porém, não é possível observação clara das fossetas, devido ao grau de dissolução da concha. No fragmento da parte posterior é possível observar ainda parte da base do septo (Fig. 7b). Além disso, as imagens em microscópio eletrônico de varredura permitem identificar as fibras de calcita (Figs. 7c, d), bem como as punctae (Fig. 7d) que tipicamente constituem as camadas internas das conchas de Bouchardia (vide Rodrigues et al. 2006).

Distribuição estratigráfica dos braquiópodes: poços Petrobras e "Sondagem de 1862" Na sucessão estudada da Bacia de Pelotas, conchas de braquiópodes não estão amplamente distribuídas na vertical. No Poço 2-PN-1-RS (Povo Novo), os braquiópodes foram encontrados no nível de 168 a 171 metros, em argilitos esverdeados. Já no Poço 2-GA-1-RS (Graxaím), as ocorrências de conchas são verificadas no nível situado a 133,40 metros da base (Fig. 2), onde ocorrem argili- 


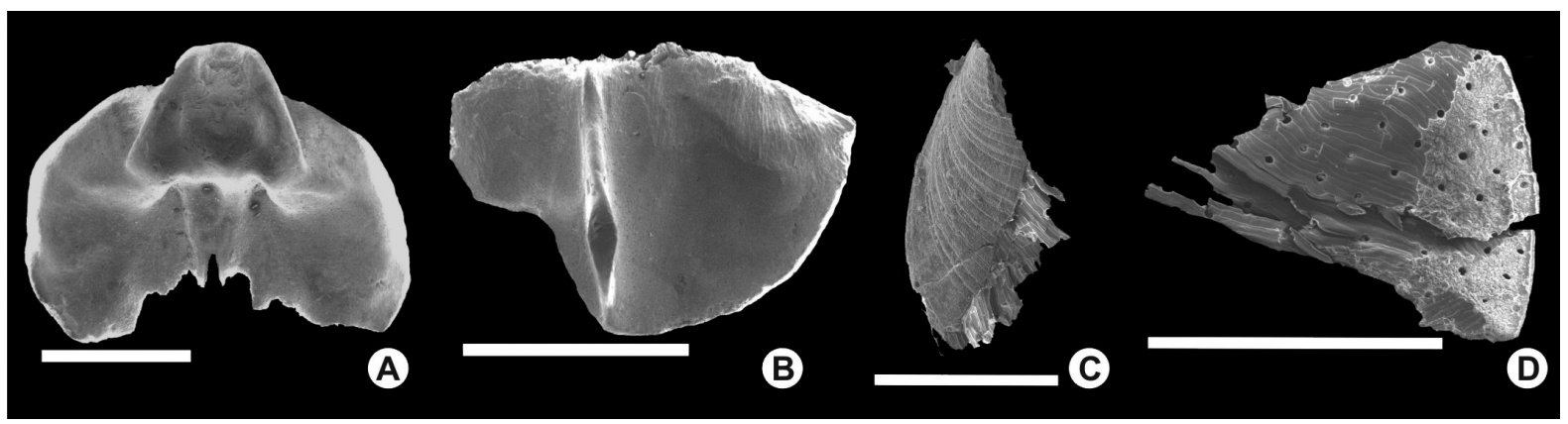

Figura 7 - Espécimes de Bouchardia sp., provenientes do poço 2-GA-1-RS. A-Valva dorsal mostrando a típica charneira de Bouchardioidea. Note a dissolução dessa estrutura, espécime DZP-18679. $B$ - Fragmento de valva dorsal, onde se observa nitidamente a presença do septo mediano, sem a ocorrência de braquidio, espécime DZP-18680. C-Fragmento de valva ventral, mostrando ao longo da fratura, a camada secundária, fibrosa, de calcita, espécime DZP-18676. D- Fragmento indistinto de concha de Bouchardia, com endopontuações (endopuncatae) e camada secundária, fibrosa, espécime DZP-18677. Escala gráfica: $2 \mathrm{~mm}$ em A e B, $400 \mu \mathrm{m} \mathrm{em} \mathrm{C,} 500 \mu \mathrm{m} \mathrm{em} \mathrm{D.}$

tos esverdeados a acinzentados, calcíferos. Por sua vez, no Poço 2-PJ-1-RS (Ponta do Juncal) as conchas estão associadas a argilitos esverdeados, que ocorrem há 123 metros (Closs 1970, Sanguinetti 1980). Em todos os casos, as conchas ocorrem associadas à Zona de Henryhowella evax (Fig. 2) (Sanguinetti 1980).

Em adição às conchas acima, valvas de $\mathrm{Bou}$ chardia foram recuperadas também, conforme já dito, no testemunho da "Sondagem de 1862". Nesse caso, os exemplares provêm de uma camada de coquina, conglomerado e brecha, com matriz lamosa, situada a 93,5 metros de profundidade (Fig. 7).

\section{DISCUSSÃO}

Tafonomia dos braquiópodes, poços 2-PN-1-RS e 2-GA-1-RS No geral, todas as conchas encontradas em argilitos, nos poços estudados, apresentam-se alteradas por dissolução (Figs. 6, 7). Tais conchas mostram, comumente, textura superficial granular, com individualização dos cristais de calcita e corrosão das estruturas proeminentes, tais como o processo cardinal, dentes, margens, umbo e bico etc. (Simões et al. 2004, Rodrigues 2006). Algumas conchas estão recristalizadas (Fig. 5d).

Um aspecto curioso é o de que, nas amostras estudadas, há um tendenciamento à preservação das valvas dorsais. Estudos experimentais de Rodrigues et al. (2006), realizados com conchas de Bouchardia rosea em meio ácido, indicam que as valvas ventrais dissolvem mais rapidamente do que as dorsais. Isso porque as valvas ventrais são comparativamente menos espessas, apresentando maior densidade de punctae. Em outras palavras, assembléias fósseis contendo conchas de Bouchardia submetidas à intensa dissolução preservam preferencialmente as valvas dorsais. Essa característica tafonômica pode ajudar a explicar o tendenciamento observado nas conchas de braquiópodes dos poços estudados.

Implicações paleoceanográficas Um tema paleogeográfico, altamente controvertido, diz respeito ao es- tabelecimento da Corrente das Malvinas (um prolongamento da Corrente Circum-Polar Antártica), durante o Mioceno. Segundo alguns autores (e.g., Boltovskoy 1979), nesse intervalo de tempo, a Passagem de Drake não estava ainda aberta e, portanto, a Corrente das Malvinas ainda não existia ou não tinha influência sobre as regiões de latitudes mais baixas, como a plataforma do Rio Grande do Sul. A questão, porém, não é tão simples. Em primeiro lugar, a Passagem de Drake foi formada pela ruptura de uma zona de subducção, que culminou com a formação da Microplaca Scotia (Livermore et al. 2004). Assim, a abertura envolveu múltiplas passagens, primeiramente de águas superficiais. Em segundo lugar, diferentes evidências e ferramentas de natureza geológica, geofísica, paleontológica e geoquímica, para citar as principais, têm sido empregadas para detectar e dar dimensão temporal aos eventos relativos à abertura da passagem de Drake e ao estabelecimento da Corrente Circum-Polar Antártica (CCA). Diferentes ferramentas fornecem dados relativos a distintos regimes de fluxos (Lyle et al. 2007). Conseqüentemente, as evidencias são, muitas vezes, divergentes. Por exemplo, reconstruções fundamentadas em dados geotectônicos indicam que a Passagem de Drake formou-se no Eo-oligoceno (cerca de $32 \mathrm{Ma}$, Lawver \& Gahagan 2003), no Neooligoceno (24 Ma, e.g. Livermore et al. 2004, Pfuhl \& Mc Cave 2005) ou no Eomioceno (23-17 Ma, Barker 2001). Já para Crame (1999), a abertura da passagem de Drake foi um processo longo, abrangendo o lapso de tempo da ordem de 37 a 23,5 +/- 2,5 Ma.

A presença de cristas no assoalho oceânico e fragmentos de crosta continental, remanescentes da evolução da "Zona de Fratura Shackleton" (no Mar de Scotia), porém, teriam bloqueado a passagem de correntes até o início do Mioceno (20-22 Ma, Barker \& Burrell 1977, Lear et al. 2000, Eagles \& Livermore 2002). Pesquisas mais recentes (Lyle et al. 2007), entretanto, indicam que a CCA estabeleceu-se no Neooligoceno (25-23 Ma), tendo aumentado sua influência 
no Eomioceno (Lyle et al. 2007). Após a abertura da Passagem de Drake houve o estabelecimento da Corrente das Malvinas, que se move em sentido oposto (sul-norte) a Corrente do Brasil (norte-sul).

Camacho (1967) e del Río (1990) reportaram que a ocorrência de associações faunísticas de águas quentes, na extremidade sul do continente americano, há aproximadamente 10 Ma pode ser explicada, assumindo-se que a Corrente das Malvinas não estava plenamente operante, no início do Neomioceno. Conseqüentemente, a proto-corrente do Brasil, de águas quentes, tinha forte influência até as altas latitudes do continente sul-americano (Martinez \& del Rio 2002). Do mesmo modo, a presença de foraminíferos tropicais/subtropicais no intervalo miocênico da Bacia de Pelotas, caracteriza a influência de condições de águas marinhas quentes (Coimbra et al. no prelo). Esses dados estão de acordo também com a presença de conchas de Bouchardia. Trata-se de um braquiópode, de afinidades com águas quentes, tropicais/subtropicais (Tommasi 1970, Manceñido \& Griffin 1988, Kowalewski et al. 2002, Simões et al. 2004). De fato, a partir do Cretáceo-Paleoceno e Mio-Plioceno, espécies desse gênero mostram um progressivo deslocamento (migração) para o norte, a partir de latitudes mais altas (Antártica e Patagônia), atingindo, no Holoceno, a plataforma dos estados do Paraná, São Paulo e Rio de Janeiro (Tommasi 1970, Manceñido \& Griffin 1988, Kowalewski et al. 2002, Simões et al. 2004).

CONCLUSÕES (1) Segundo a revisão aqui realizada, os braquiópodes encontrados no testemunho da "Sondagem de 1862", pertencem à espécie Bouchardia transplatina e não Bouchardia cf. zitteli, como anteriormente suposto. $\mathrm{O}$ equivoco taxonômico verificado em Martins (1952) decorreu, provavelmente, do estado de preservação das amostras disponíveis para estudo, ou seja, as conchas da "Sondagem de 1862". Essas estão claramente dissolvidas, com nítida mudança na forma geral da concha;

(2) Os exemplares provenientes dos poços 2-PN-1-RS e 2-GA-1-RS, recuperados de material de calha, pertencem ao gênero Bouchardia. Sua identificação precisa, ao nível específico, fica prejudicada, contudo, em razão da intensa corrosão das conchas, que eliminou feições anatômicas proeminentes (e.g., dentes) e modificou a morfologia geral das valvas, por dissolução das margens.

(3) A presença de bouchardídeos na sucessão miocênica (Zona de Henryhowella evax), da Bacia de Pelotas, corrobora a influência de águas plataformais tropicais/subtropicais, nessa porção do continente sulamericano, durante o Mioceno, reforçando a idéia de que, nesse intervalo de tempo, a Passagem de Drake não estava plenamente estabelecida, bem como a Corrente das Malvinas não era ainda ativa.

Agradecimentos Os autores gostariam de agradecer ao Departamento de Geologia Sedimentar e Ambiental, IGc/USP, pelo empréstimo dos exemplares de Bouchardia zitteli, provenientes da Formação San Julián, Patagônia, Argentina; ao Sergio Martinez (Instituto Nacional de Geologia y Paleontologia, Facultad de Ciências, Uruguai), pelo empréstimo dos exemplares de Bouchardia transplatina. Ao Museu Nacional, RJ, em especial a Vera Maria Medina da Fonseca, pelo acesso aos fósseis dos testemunhos da "Sondagem de 1862". Ao Luiz Henrique Cruz de Mello, pela confecção dos moldes das conchas da referida sondagem. O presente estudo é uma contribuição aos projetos FAPESP 06/00169-1, 06/59416-8, 06/04777-6, e CNPq 306601/2006-0, 473971/2006-2, 305537/2006-7.

\section{Referências}

Barker P.F. 2001. Scotia Sea regional tectonic evolution: implications for mantle flow and paleocirculation. Earth Sc. Rev., 55:1-39.

Barker P.F. \& Burrell J. 1977. The opening of Drake Passage. Mar. Geol., 25:15-34.

Boltovskoy E. 1979. Paleoceanografia del Atlántico Sudoccidental desde el Mioceno, Ameghiniana, 16:3-4.

Camacho H.H. 1967. Las transgresiones del Cretacico Superior y Terciario de la Argentina. Rev. Asoc. Geol. Argent., 22:253-280.

Carreño A.L., Coimbra J.C., Sanguinetti Y.T. 1997. Biostratigraphy of the Late Neogene and Quaternary ostracodes in the Pelotas Basin, Southern Brazil. Gaia, 14:33-43.

Carreño A.L., Coimbra J.C., Carmo D.A. 1999. Late Cenozoic sea level changes evidenced by ostracodes in the Pelotas basin, southernmost Brazil. Mar. Micropaleontol., 37:117-129.

Closs D. 1967. Miocene Planktonic foraminifera from south- ern Brazil. Micropaleont., 13:337-344.

Closs D. 1970. Estratigrafia da Bacia de Pelotas, Rio Grande do Sul. Iheringia (Série Geologia), 3:3-37.

Coimbra J.C., Carreño A.L., Anjos-Zerfass G.S.A. (no prelo). Biostratigraphy and paleoceanographical significance of the Neogene planktonic foraminifera from the Pelotas Basin, southernmost Brazil. Rev. Micropaleont. (aceito, sem data de publicação).

Crame J.A. 1999. An evolutionary perspective on marine faunal connections between southernmost South America and Antarctica. Scientia Marina, 63(supl.1):1-14.

del Río C.J. 1990. Composicion, origen y significado paleoclimatico de la malacofauna 'Entrerriense' (Mioceno medio) de la Argentina. An. Acad. Nac. Cs. Ex., Fis. Nat. Buenos Aires, 42:205-224.

del Río C.J. 2004. Revision of the Neogene giant pectinids of Eastern Santa Cruz and Chubut provinces (Patagonia - Argentina). J. Paleont., 78:690-699.

Dias J.L., Silveira D.P., Sad A.R.E., Latgé M.A.L. 1994. Ba- 
cia de Pelotas: Estágio atual do conhecimento geológico. In: Bol. Geoc. Petrobras, 8:235-245.

Eagles G. \& Livermore R.A. 2002. Opening history of Powell Basin, Antarctic Peninsula. Mar. Geol., 185:195-205.

Fernandes J.M.G. 1975. O gênero Uvigerina (Foraminiferida) do Cenozóico Superior na Bacia de Pelotas, Rio Grande do Sul, Brasil. Dissertação de Mestrado, Instituto de Geociências, Universidade Federal do Rio Grande do Sul, 121p.

Fontana R. L. 1996. Geotectônica e sismoestratigrafia da Bacia de Pelotas e Plataforma de Florianópolis. Tese de Doutorado, Instituto de Geociências, Universidade Federal do Rio Grande do Sul, 214 p.

Gliesch R. 1932. Sobre a origem dos sambaquis. Egatéa, 17:199-207.

Gomide J. 1989. Bacia de Pelotas: biocronoestratigrafia baseada em nanofósseis calcários. In: SBP, Congresso Brasileiro de Paleontologia, 11, Curitiba, Anais, p. 338351.

Ihering H. von. 1903. Lês brachiopodes tertiaires de Patagonie. An. Mus. Nac. Buenos Aires, ser. III, 2:321-348.

Kowalewski M., Simões M.G., Carroll M., Rodland D.L. 2002. Abundant brachiopods on a tropical, upwellinginfluenced shelf (Southeast Brazilian Bight, South Atlantic). Palaios, 17:274-283.

Lawver L.A. \& Gahagan L.M. 2003. Evolution of Cenozoic seaways in the circum-Antarctic region. Palaeogeog., Palaeoclimatol., Palaeoecol., 198:11-37.

Lear C.H., Elderfield H., Wilson P.A. 2000. Cenozoic deepsea temperatures and global ice volumes from $\mathrm{Mg} / \mathrm{Ca}$ in benthic foraminiferal calcite. Science, 287:269-272.

Livermore R., Eagles G., Morris P., Maldonado A. 2004. Shackleton Fracture Zone: no barrier to early circumpolar ocean circulation. Geology, 32:797-800.

Lyle M., Gibbs S., Moore T.C., Rea D. K. 2007. Late Oligocene initiation of the Antartic Circumpolar Current: evidence from the South Pacific. Geology, 35:691-694.

Madeira-Falcetta M., Thiesen Z.V., Kotzian S.B., Godolphim M. 1980. Interpretação de dois testemunhos de sondagem (PSD-3 e ALB) na planície costeira do Rio Grande do Sul, Brasil. Pesquisas, 13:91-107.

Manceñido M.O. \& Griffin M. 1988. Distribution and palaeoenvironmental significance of the genus Bouchardia (Brachiopoda, Terebratellidina): its bearing on the Cenozoic evolution of the South Atlantic. Rev. Bras. Geoc., 18:201-211.

Martinez S. 1994. Bioestratigrafía (Invertebrados) de la Formación Camacho (Mioceno, Uruguay). Tese de Doutoramento, Universidad de Buenos Aires, Facultad Ciencias Exactas y Naturales, 346p.

Martinez S. \& del Río C.J. 2002. Late Miocene Molluscs from the Southwestern Atlantic Ocean (Argentina and Uruguay): a palaeobiogeographic analysis. Palaeogeog., Palaeoclimatol., Palaeoecol., 188:167-187.

Martins E.A. 1952. Fósseis da sondagem de 1862 em Pelotas, Estado do Rio Grande do Sul. Bol. Mus.Nac. Geol. n.s., 17:1-8.

Mello L.H. 2004. Análise cladística dos Bouchardiinae Allan, 1940 (Brachiopoda, Terebratellidae): implica- ções sistemáticas e paleozoogeográficas. Tese de Doutoramento, Instituto de Geociências, Universidade de São Paulo, 181p.

Milani E.J., Brandão J.A.S.L., Zalán P.V., Gamboa L.A.P. 2000. Petróleo na margem continental brasileira: geologia, exploração, resultados e perspectivas. Brazilian $J$. Geophysics, 18:351-396.

Mohriak W.U. 2003. Bacias Sedimentares da Margem Continental Brasileira. In: Bizzi L.A., Schobbenhaus R.M. Vidotti, Gonçalves J.H. (eds.) Geologia, Tectônica e Recursos Minerais do Brasil. CPRM, p. 87-94.

Pfuhl H.A. \& McCave I.N. 2005. Evidence for late Oligocene establishment of the Antarctic Circumpolar Current. Earth and Planetary Science Letters, 235:715-728.

Rodrigues S.C. 2006. Tafonomia de moluscos bivalves e braquiópodes das enseadas de Ubatuba e Picinguaba, norte do estado de São Paulo: implicações do uso de assinaturas tafonômicas no reconhecimento de gradientes ambientais. Tese de Doutoramento, Instituto de Geociências, Universidade de São Paulo, 118p.

Rodrigues S.C., Neves J., Simões M., Kowalewski M. 2006. Dissolution of Bouchardia rosea shells: implications to taphonomic bias in Rhynchonelliform brachiopod assemblages. In: Congreso Argentino de Paleontologia, 9, Resúmenes, p. 266.

Sanguinetti Y.T. 1979. Miocene ostracodes of the Pelotas Basin, state of Rio Grande do Sul, Brasil. Pesquisas, 12:119-187.

Sanguinetti Y.T. 1980. Bioestratigrafia (ostracodes) do Mioceno da Bacia de Pelotas, Rio Grande do Sul. Pesquisas, 13:7-34.

Sanguinetti Y.T., Ornellas L.P., Coimbra J.C. 1991. PostMiocene ostracodes from the Pelotas basin suothern Brazil, Taxonomy - Part I. Pesquisas, 18:138-155.

Sanguinetti Y.T., Ornellas L.P., Coimbra J.C., Ramos M.I.F. 1992. Post-Miocene ostracodes from the Pelotas basin suothern Brazil, Taxonomy - Part II. Pesquisas, 19:155166.

Simões M.G., Kowalewski M., Mello L.H.C., Rodland D.L., Carroll M. 2004. Present-day terebratulid brachiopods from the southern Brazilian Shelf: Paleontological and biogeographic implications. Palaeontology, 47:515533.

Thiesen Z.V. 1975. Bolivinitidae e Caucasinidae (Foraminiferida) do Cenozóico superior da Bacia de Pelotas, Rio Grande do Sul, Brasil. Dissertação de Mestrado, Instituto de Geociências, Universidade Federal do Rio Grande do Sul, 99p.

Tommasi L.R. 1970. Sobre o braquiópode Bouchardia rosea (Mawe), 1823. Bol. Inst. Oceanog., 19:33-42.

White I.C. 1908. Relatório final da Comissão de Estudos das Minas de Carvão de Pedra do Brasil. Rio de Janeiro: DNPM, 1988. Parte I, p.1-300; Parte II, p. 301-617.

Manuscrito ID 11948 Submetido em 31 de julho de 2008 Aceito em 12 de dezembro de 2008 Sistema eletrônico de submissão 